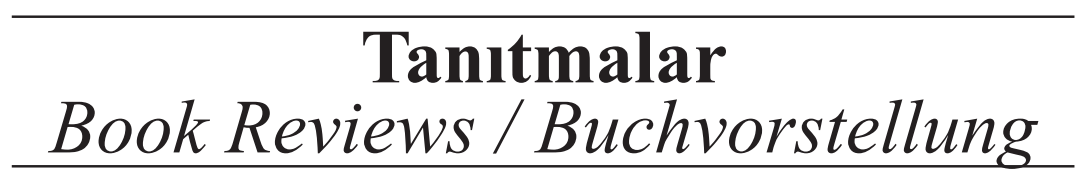



Mehmet Yaman (Dede) (2018). Türk Modernleşmesinde Bir Alevi Ocakzade Dinbilimcinin Seyir Defteri. Haz. Ali Yaman. Köln: Alevi-Bektaşi Kültür Enstitüsü Yayınları.

\section{Arslan KILIÇ}

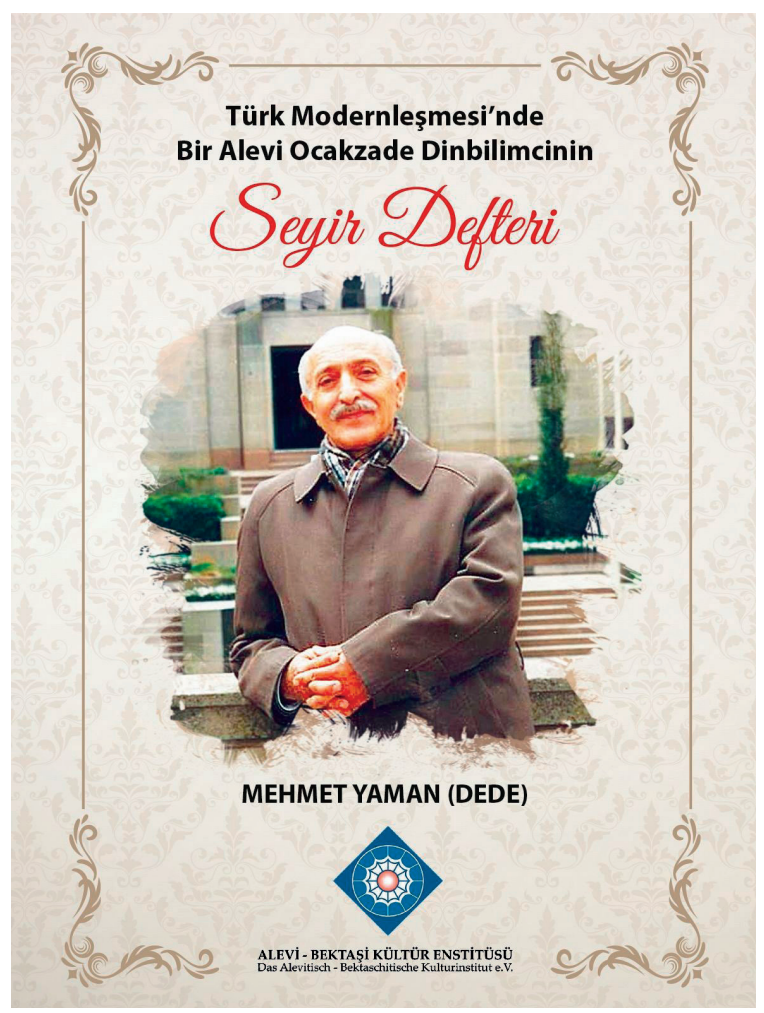

Mehmet Yaman Dede zählt zu den von der Alevitsch-Bektaschi Gemeinde hervorgebrachten angesehenen Geistlichen (mürşid) und Auszüge seiner Lebenserinnerungen aus seinen lebenslänglich geführten Tagebüchern wurden seitens des Alevitisch-Bektaschi Kulturinstituts veröffentlicht. Mehmet Yaman Dede, der über Sprachkenntnisse in Arabisch, Persisch und Englisch verfügte, ging nach 30

* Arş. Gör., İzmir Kâtip Çelebi Üniversitesi, SBBF, Tarih Bölümü, İzmir, arslan.kilic@ikc.edu. tr. 
Jahren Dienst auf dem Bildungssektor in die Rente und gab ungefähr 20 Jahre lang (von 1990 Jahren bis in die 2010'er Jahre) weltweit Seminare über das Alevitentum und nahm an Podiumsdiskussionen und Konferenzen teil. Er setzte seine Dienste bis zu seinem Tod am 8. Januar 2014 fort.

Mehmet Yaman Dede versuchte schon zu seinen Lebzeiten aus seinen Lebenserinnerungen ein Buch zu formen, wozu es aber aus gesundheitlichen Gründen nicht mehr kam, so dass dieses vorliegende Buch von seinem Sohn Prof. Dr. Ali Yaman nach einer gründlichen Vorbereitungsphase herausgebracht wurde. Die Lebenserinnerungen von Mehmet Yaman Dede spielen wegen ihrer Beiträge zur Aufklärung der religiösen Dimension der türkischen Modernisierungsgeschichte eine bedeutende Rolle. Zudem sind Tagebücher immer als wichtig anzusehen, weil sie zum Verständnis vergangener Ereignisse, über die wenig bekannt ist, beitragen. Viele Wahrheiten, die von den dominierenden politischen, religiösen oder ökonomischen Kreisen vertuscht werden, können mittels veröffentlichter Tagebücher die Aufmerksamkeit der Öffentlichkeit erlangen.

Prof. Yaman, Herausgeber des Buches, fasst die ganze Prozedur zur Buchveröffentlichung wie folgt zusammen: ,... Ich möchte an dieser Stelle, wenn auch nur kury, auf die Entstehungsgeschichte dieses vorliegenden Buches eingehen, welches Sie gerade lesen. Ursprünglich sollte das Buch in den Jahren 2012-2013, also noch zu Lebzeiten meines Vaters veröffentlicht werden. Eine Reihe von Entwicklungen hat aber diesen Termin unmöglich gemacht und das Buch konnte erst nach dem Tode meines Vaters erscheinen. Der erste Grund, warum das Buch in den Lebzeiten meines Vaters nicht erscheinen konnte, hängt wohl damit zusammen, dass mein Vater an Herrn Murat Korkmaz, Eigentümer des Verlagshauses Horasan Yaynlar, eine Zahlung für die Satzkosten geleistet hatte, dieser Herr aber sowohl den Computersatz des Buches als auch einige der sehr wertvollen Bilder verloren hat. Mein Vater war über diesen Umstand sehr traurig. Dadurch hat sich die Veröffentlichung des Buches für zwei bis drei Jahre bingezogen. Alsdann sogar die von meinem Vater sehr geschätzten Herren Onarl Celal Dede und Ayban Aydin den Verleger Murat Korkmaz, besuchten und ibn um die Herausgabe des Satzes und der Bilder baten, wurden ihre Bemühungen nicht belohnt. Ich selbst habe diesen Verleger auch telefonisch kontaktiert und dann erfahren müssen, dass viele weitere Menschen ebenfalls ähnliche Probleme mit dem Verleger gehabt haben.

In dieser Phase wurde bei meinem Vater eine sehr schwere Lungenerkrankung diagnostiziert und man begann damit, ihn mit Chemotherapie zu behandeln. Wir alle waren sehr traurig darüber, aber er war es, der unter den größten Schmerzen litt. 
Ich habe dann die Texte, die mein Vater über die Schlacht Kerbela verfasst hat, mit einigen meiner Schriften darüber zusammengestellt und diese dann im Computer bearbeitet und daraus ein Buch über das Kerbela-Ereignis vorbereitet. Als ich meinen Vater im Krankenhaus besuchte, fragte ich mich, ob es zur Linderung seiner Schmerzen beitragen würde, wenn ich ibn darüber in Kenntnis setzen würde. Seit meiner Kindheit wusste ich nämlich zu gut, dass er jeden Tag mit seinen Büchern, mit Schreiben und Lesen beschäftigt war.

In der Hoffnung, ibn mit dieser Nachricht psychologisch aufzubauen, beschloss ich, tätig ₹u werden und sprach dann mit Herrn Engin darüber, damit seine Lebenserinnerungen vom dem Verlagshaus Nokta Yaymlar verlegt werden. Ich kann es aber bis heute immer noch nicht vergessen, als ich mit meinem Vater und mit meiner Mutter zu Hause frühstückte und währenddessen ein Mitarbeiter des Verlagshauses zu uns nach Hause kam, um seine Notizen für die Verlegung des Buches mitzunehmen und mein Vater daraufhin sagte, dass er vor habe, gewisse Ergänzungen zu machen und dann darauf verzichtete, das Buch setzen zu lassen.

Später wurde seine Krankheit noch intensiver und nach einer sehr schmerzhaften Periode verstarb unser Vater im Jahre 2014, des Monats Januar. Er wurde nach einem Gottesdienst in Yenibosna Cemevi in Istanbul und nach einem feierlichen Tranerakt, der in unserem Dorf in dem Hidir Abdal Sultan Ocak stattfand, beigesetzt.

Die auserwählten Abschnitte aus seinen handgeschriebenen Lebenserinnerungen bestehen aus 666 Seiten mit DIN A4 Format. Da es nicht einfach schien, seine Handschrift zu entziffern, habe ich zunächst versucht, die handschriftlichen Notizen computerlich zu erfassen. In Wirklichkeit gibt es aber noch vielmehr handgeschriebene Notizen jenseits seiner bier berücksichtigten Lebenserinnerungen, die, sofern sie geordnet werden mögen, als Buch erscheinen könnten. Da die Zusammenstellung der Auszüge aus den Notizen meines Vater für mich sehr viel Zeit in Anspruch nehmen würde, hat Herr Ufuk. Altmer von der Druckerei Ufuk. Matbaasi, der meinen Vater auch sebr gut kannte und der auch in der Vergangenheit all seine Bücher gesetzt und gedruckt hatte, dieses vorliegende Buch ebenfalls gesetzt.

Die Frage, ob das Buch von einem Verlagshaus oder von einer Alevi-Bektaschi Einrichtung berausgegeben werden sollte, war auch von Bedeutung. Während eines Gesprächs darüber mit Frau Gülizar Cengiz, der angesehenen Leiterin des Alevi-Bektaschi Kulturinstituts, wo ich auch ein Mitglied des Wissenschaftsbeirats bin, hat mir Fran Gülizar gesagt, dass das Kulturinstitut es sehr schätzen würde, das Tagebuch eines so hochangesehenen und intellektuellen geistlichen Oberhauptes wie Mehmet Yaman Dede herauszugeben, der aufgrund seiner Geistlichen-Abstammung (Ocak), 
seines Dorfes, seiner Ausbildung und seiner erwiesenen Dienste über einen besonderen Platz in der Geschichte des Alevitentums verfügt.

Mein hochseliger Vater kannte Frau Gülizar Cengiz und Ihren Gatten Hasan Cengiz seit Jahren. Schwester Gülizar kam auch in den letzten Stadien der Erkrankung meines Vaters zu ihm in die Wohnung, um ihn zu besuchen und während einer freundschaftlichen Unterhaltung hat mein Vater seine Bibliothek dem Alevi-Bektaschi Kulturinstitut gestiftet. Nach Abschluss des Setzvorgangs des Buches habe ich auch innerhalb von 2-3 Monaten die schriftliche Abfassung und Lesung von einigen unvollständigen Notizen beendet. Es hat auch Zeit gekostet, das Bildmaterial zusammenzustellen. Unterdessen wurde ich von dem von meinem seligen Vater hochgeschätzten Herrn Ayhan Aydin in Kenntnis gesetzt, dass er einige Bilder, die er selbst gemacht hat, zur Verfügung stellen kann. Ich habe das Buch mehrfach Korrektur gelesen, um die Satafehler zu beheben. Das Buch ist dankenswerterweise auch von dem Assistenzprofessor Herrn Dr. Mehmet ERSAL Korrektur gelesen worden.

Soweit zur Geschichte der Vorbereitung der Buchveröffentlichung. Wichtig ist jedoch die Lebensgeschichte eines Menschen, um die es hier geht. Es nahm so viel Zeit in Anspruch, ein Buch über ein Leben zu schreiben, es zu setzen und zu veröffentlichen, während die Lebenserfahrungen, die er machte, mehr als siebzig Jahre umfassten. Mein Vater hatte das Vorwort des Buches bereits angefangen, es aber nicht zu Ende geschrieben und in seinen Notizen erwähnte er, dass er das Vorwort nach Beendigung des Setzvorgangs zu Ende schreiben würde. Außerdem musste ich noch einige Themen, deren Setzvorgang bereits abgeschlossen war und einige, die noch gesetzt werden mussten, einreiben. Daher lag es an mir, darüber zu berichten. Auf dieser Weise kam das Buch heraus. Es war auch sehr angebracht, dass das Buch durch das Alevitisch-Bektaschi Kulturinstitut herausgegeben wurde, welches sich durch akademische Studien auf dem Gebiet des Alevitentums auszeichnet und eine internationale Forschungszeitschrift mit Gutachterrat herausbringt. Wenn man die Notizen meines seligen Vater liest, sieht man, dass die Liebe, die man zur Sache des Alevitentums-Bektaschitums empfindet, selbst unter den schwierigsten Bedingungen möglich ist... , (S. 15-17)

Das vorliegende Buch umfasst 352 Seiten und ist bebildert mit farbigen Fotos und Dokumenten, die auf die Lebenserinnerungen von Yaman Dede hinweisen. Am Anfang des Buches ist eine Einführung zu lesen, welches von Frau Gülizar Cengiz, der Leiterin des Alevitsch-Bektaschi Kulturinstituts stammt. Dahinter kommen weitere Texte von alevitischen Geistlichen (pir) wie Mustafa Düzgün Dede, Muharrem Ercan Dede, Yüksel Bilgin Dede, die den verstorbenen Yaman Dede kannten, gefolgt von weiteren kurzen Bewertungen von ForscherInnen des Alevitentums wie Prof. Dr. 
Raoul Motika, Uni.-Doz. Dr. Markus Dressler, Uni.-Doz. Dr. Robert Langer und Dr. David Shankland. Diese Bewertungen, die auf der Rückseite des Umschlags zu lesen sind, möchten wir auch vorstellen:

Mubarrem Ercan (Dede), Leiter des Karaca Abmet Sultan Dergah (Türkei): Unser Mehmet Yaman Dede war einer der ersten Gründer des Karaca Abmet Sultan Dergah, er war der Sohn von Hidir Abdal Sultan. Wir gedenken seiner in voller Dankbarkeit für die Dienste, die er für die Alevi-Bektaschi Gemeinschaft geleistet hat. Die Sorgen, von denen er in seinen Lebenserinnerungen berichtet hat, haben wir als Aleviten immer wieder erlebt und wir erleben es immer noch. Mit den Werken, die er hinterlassen hat, mit seinen Lebenserinnerungen wird er der alevitischen Gesellschaft weiterhin dienen. Es macht uns außerordentlich traurig, dass er in dem Moment, in dem er noch der Gemeinschaft soviel geben könnte, von uns gegangen ist. Möge seine Seele frob und glücklich sein, möge er ein Heim im Paradies finden. Möge Gott seinem Sohn Professor Ali Yaman, der wie sein Vater dem selben Weg „Gott, Mubammed, Ali“folgt, Gesundheit, Woblbefinden, Frieden bescheren, damit er den selben Weg und die gleichen Regeln fortsetzen kann. Mit inständiger Bitte um Liebe (Assk-ı niyazlarmla).

Mustafa Dürgün Dede, Gründungsvorsitzender der Alevitischen Akademie (Schweden): Mehmet Yaman Dede war ein weiser Mensch, der sich auf vielen Gebieten Bildung verschafft hat. Er war eine Musterpersönlichkeit mit einer sehr großen Lebensperspektive und mit großer Toleran₹: Ich grüße alle ganz heralich, die sein Andenken und seine Werke bewahren.

Yükesel Bilgin (Dede), ALEVI Generalvorsitzer (Österreich): Unser hoch verehrter Mehmet Yaman Dede war in einer Zeit, in der sich das Alevitentum in der Anfangsphase seiner Institutionalisierung in Europa befand, ein sehr weiser Pädagoge, der allen voran in der Türkei, in Deutschland und Österreich und in vielen anderen europäischen Ländern unsere Gesellschaft mit seinen Werken, Seminaren, Konferenzen, Glaubensdiensten aufgeklärt hat. Aufgrund seiner großen Verdienste werden wir ihn als Alevitische Gemeinschaft sehr vermissen und stets seiner gedenken. Er, der unser geistige Führer war, wird auch nach seinem Tode mit seinen Werken und Lebenserinnerungen weiterwirken. Es ist für uns Aleviten eine große Ehre, dass wir so einen geistigen Führer wie Mehmet Yaman Dede haben, der den Alevitischen Weg so fortgesetžt hat, wie wir dies auch seit Jahrhunderten von anderen geistigen Führern kennen. Als eine alevitische Einrichtung, die sich stark bemüht, die alevitische Gemeinschaft in Österreich in die nächsten Jabrhunderte zu bringen, verbengen wir uns vor seinen Diensten, die er für unseren guten Weg geleistet hat. Möge seine Seele froh und glücklich sein. 
Uni.-Doz: Dr. Markus Dressler, Leipzig Universität (Deutschland): Dieses Buch, in dem die Lebenserinnerungen von Mehmet Yaman Dede zusammengestellt wurden, beleuchtet nicht nur die bistorischen und traditionellen Dimensionen des Alevitentums, es beleuchtet auch seine Vitalisierung und seine politischen Probleme aus der jüngsten Vergangenheit. Dieses Buch porträtiert sowohl einen republikanischen Intellektuellen, der sein Land und sein Volk liebte als auch die Seele eines selten feinen Menschen, der das traditionelle Alevitentum mit seiner Sprache und mit seiner Persönlichkeit repräsentiert hat. Diese Lebenserinnerungen, die aus verschiedenen Phasen der Türkischen Republik bestehen, präsentieren dem Leser eine einzigartige Perspektive, die vermittelt, was es politisch und gesellschaftlich bedeutet, angesichts einer Sunnitischen Mebrheit Alevitisch zu sein und dass man ein Theologiestudium absolvieren kann obne sein Alevitentum zu leugnen und dass man sein traditionelles Alevitentum gemäß den Erfordernissen und Bedürfnissen der modernen Zeit interpretiert. Diese Texte haben einen Dokumentencharakter, bei denen es sich nicht nur um das Vermächtnis eines alevitischen Geistlichen handelt, sie sind zugleich auch wie ein Spielgel der republikanischen Periode der Türkei.

Dr. David Shankland, Royal Anthropological Society (England): Kurz nach dem ich meine Untersuchungen über die Aleviten in Anatolien begann, babe ich erfahren, wie wichtig die Geistlichenhäuser (Ocaklar) im Alevitentum sind. Es ist angebracht zu sagen, dass die dede ocaklar sowobl moralisch als auch geistig das Herz des Alevitentums bilden. Persönlichkeiten, welche die Geistlichen-Würde (dedelik makami) genießen, sind dermaßen bedeutend, dass deren Ansehen sich ständig vermehrt und ihr Ruhm weit über ihr Umfeld binaus bekannt ist. Mehmet Yaman Dede, dessen Lebenserinnerungen in diesem Buch veröffentlicht werden, gehört ebenfalls zu den weisen Persönlichkeiten, dessen Ansehen sich ständig vermehrt hat. Er war unter meinen alevitischen Freunden als ein weiser, höflicher, großä̈giger Mensch bekannt, der der Träger der besten Eigenschaften der Geistlichen-Würde war und deren Ideal er voll vorlebte. Es ist für mich ein besonderes Privileg zu sagen, diesen ebrenwerten Geistlichen (dede) kennenzulernen und soviel von seinen schriftlichen Werken zu lernen. Dank dieser Werke werden die zukünftigen Generationen nach dem Tod des verehrten Mehmet Dede weiterhin von seinen wertvollen Lehren profitieren.

Dozent Dr. Robert Langer, Orient-Institut (Türkei): Ich habe Mebmet Yaman Dede zum ersten Mal im Jahre 2004 kennengelernt als ich meine Forschungen über die alevitischen Rituale begann. Es war für mich ein großer Genuss so einen belesenen Aleviten wie Mehmet Yaman Dede kennenzulernen. Er war nicht nur jemand, der nur Arabisch sprach und Türkisch-Texte verstehen konnte, die mit arabischen und persischen Schriftzeichen verfasst waren, also nicht nur jemand, der in diesen Sprachen autorisiert war, er hatte auch in Istanbul modernes Persisch studiert und sich mit mir auch in dieser Sprache unterhalten. Dies bedeutet, dass er zum Verständnis des Mitteleren Ostens 
in den klassischen „drei Sprachen“" ('elsine-i selase') aktiv und passiv fähig war. Darüber hinaus war er der englischen Sprache fließend mächtig und er sprach obne Akzent und auf hohem Niveau und mit großer Freude. Als ich und meine Kollegin Frau Janina Karolewski später das Privileg hatten, das Dorf Ocak Köyü von Mehmet Yaman Dede zu besuchen, konnte er die in türkischer Handschrift geschriebenen Dokumente aus der Vergangenheit sofort lesen, die wir dabei hatten, die er zuvor aber nicht gesehen hatte. All das war bemerkenswert. Jedoch reicht das alles nicht aus, um Mehmet Yaman Dede richtig zu beschreiben. Die edelste Seite seiner Charaktereigenschaften war seine Aufgeschlossenheit für Ideen und seine Freundschaft. Die alevitische Gemeinschaft hat mit dem seligen Mehmet Dede einen großen Gelehrten, einen Geistlichen (Dede) und einen vorzüglichen Menschen verloren. Mögen Glückseligkeit und Freude sein Weg werden (Revan şad olsun).

Prof. Dr. Raoul Motika, Universität Hamburg (Deutschland): Ich börte zum ersten Mal in den neunziger Jahren von den Aktivitäten von Mehmet Yaman, dem seligen großen Geistlichen (dede), Intellektuellen, und Autor. Er leistete in diesen Jahren insbesondere große Dienste für die in Deutschland lebende alevitische Gemeinde in dem Alevitischen Kulturzentrum in Mannheim, welches sich in der Nähe meines Arbeitsplatzes an der Heidelberg Universität befand. Wir haben uns leider erst nach Jahren persönlich kennengelernt, ich hatte aber schon vorher seine sehr bedeutenden Werke mit großem Interesse gelesen. Er hat aber nicht nur wertvolle Beiträge für das Fortleben des Alevitentums unter schwierigsten Bedingungen in einer sich verändernden Welt geleistet, er trug zugleich auch sehr wertvolle Beiträge in die Religions- und Geschichtswissenschaften bei. In den folgenden Jahren konnten wir uns, wenn auch kurz, anlässlich unterschiedlicher Gelegenheiten treffen, und zwar für Forschungszwecke über das Alevitentum und in Zusammenhang damit in Konferenzen, ich war aber immer sehr beeindruckt von der aufrichtigen und hilfsbereiten Persönlichkeit von Mehmet Dede. Die längste Zeit, die wir zusammen verbracht haben, war während einer Motorradreise, die ich zusammen mit meiner Frau in Anatolien unternahm. Wir kamen zu der Zeit mit Mehmet Dede in dem Dorf Arapgir Onar Köyü zusammen und haben dann an dem von ihm selbst geleiteten alevitischen Gottesdienst (Cem rituali) teilgenommen. In späteren Tagen lud er uns in sein Heimatdorf Ocak Köy ein und er beherbergte uns in seinem Haus. Gleichwohl war es für uns eine große Ehre und Freude, als Gast bei Mehmet Dede und bei seiner Familie zu bleiben. Während dieser Tage haben wir andere Seiten der Persönlichkeit von Mehmet Dede kennengelernt und haben uns davon gründlich überzengt, über was für feinfühlige, familienorientierte und milde Eigenschaften er außerdem verfügte. Wir verbrachten eine sehr schöne Zeit miteinander. In einer Phase, in der ich ernsthaft erkerankte, wurde auch Mehmet Dede schwer krank. Er hat diese schweren Tage mit seiner ihm eigenen geistigen Würde ausgehalten. Der Tod von Mehmet Dede, den alle liebten, ist für die Dörfer, aus denen die Geistlichen stammen (Ocak-Dörfer), für die alevitische Gemeinschaft, für die Wissenschaftswelt 
und für alle Menschen ein großer Verlust. Möge er in Frieden ruhen. Wir werden ihn niemals vergessen. ,

In den Lebenserinnerungen unter dem Titel „Mein Heimatdorf Ocak", wird nicht nur über Karaca Abmet Sultan und Hidir Abdal Sultan informiert, neben diesen Schilderungen liefern die Lebenserinnerungen auch Informationen darüber, wie Mehmet Yaman Dede begann Saz. zu spielen, wie er zu musabip wurde, wie seine Erlebnisse als Schüler/Student und als Lebrer waren, wie seine erste Teilnahme an Gedenktagen an Hadschi Bektasch und Abdal Musa, war und wie seine Freundschaften zu Aşık Veysel, Aşık Davut Sulari, Ali Ekber Ciçek waren, ferner werden weitere Informationen geliefert über seine Forschungen über das Alevitentum, über seine Publikationen, seine Arbeiten im Ausland, wobei insbesondere seine Erfahrungen, die erpersönlich als Schüler/Student aufgrund der Diskriminierung von Konfession erlebt hat, eine zentrale Rolle in den Lebenserinnerungen spielen und seine Darstellungen, die auf Berichte von Zeugenaussagen basieren, die schilderten, wie intensiv und systematisch der Sunnitische Doktrinarismus in der Türkei gegen die Aleviten sogar in den religiösen Bildungseinrichtungen fortgeset?t wurde. Die verschiedenen Kapiteln des Buches weisen mit Nachdruck darauf bin, dass nach seiner Meinung der Frieden und das Woblbehagen in der Türkei nur dann möglich ist, wenn das Land sich vom konfessionellem Sektierertum befreit.

Mehmet Yaman Dede erklärt in dem von ihm selbst verfassten Vorwort, wie und warum er aus seinen Lebenserinnerungen ein Buch geformt hat mit folgenden Worten: "Meine geschätzten Leser, ich habe versucht, in MEINEN LEBENSERINNERUNGEN unparteiisch zu sein, keinen Lug und Trug zu verbreiten und nur die Wahrheit zu schreiben und ohne Zensur in einer offenen Art über gute und schlechte Ereignisse zu berichten, die ich während meiner Kindheit, Jugend, Studentenzeit, Lehrertätigkeit, Militärdienstzeit und in anderen Lebensabschnitten persönlich erlebt habe und die ich auch selbst bezeugen kann, damit die türkische Gesellschaft darüber in Kenntnis gesetzt wird und ich dadurch meinem Volk und der Menschheit einen Dienst erweisen kann und wir in der Periode der Republik Türkei genauso aufgeklärt werden ie in der Osmanischen Zeit und damit - und dies ist meine Absicht - diejenigen „Parasiten der Religionsfanatiker“, die giftiger sind als das Gift selbst, welche uns auch daran hindern, das Niveau der fortschrittlichen Nationen zu erreichen, festgestellt werden und die nötigen Maßnahmen gegen diese seitens unseres Staates und unserer BürgerInnen ergriffen werden mögen. Ich glaube daran, dass meine gewissenhaften LeserInnen mir zustimmen werden, denn ich fühle 
mich gezwungen, über alle Ereignisse zu schreiben, die ich in meinem siebzig Jahre umfassenden Leben gesehen und erlebt habe.

Wie Sie in den nachfolgenden Seiten auch lesen werden, gab es neugierige Leute, die mich ernsthaft danach fragten, warum ich, der doch ungewollt sunnitische Theologie studiert hat, immer noch nicht assimiliert wäre (denn alle alevitischen Kinder, die dort studierten, wurden assimiliert) und diese Leute hegten zurecht Misstrauten gegen mich und Hunderte Aleviten nahmen sogar an, dass ich inzwischen zum Sunnitentum konvertiert wäre, und zwar dermaßen, dass sogar Onarlı Dr. İsmail Kaygusuz Can, den ich in London (in März-2006) persönlich traf, mir gegenüber Folgendes sagte (nach dem er wohl gehört hatte, dass meine ganze Lebenszeit nicht dafür ausgereicht hätte, jedem einzelnen diesbezüglich eine separate Antwort zu geben und ich über diese Dinge umfassend in meinen Lebenserinnerungen berichten werde): ,.... Mehmet Dede, aufgrund deines Studiums verdächtigen dich die Leute, ein Sunnit geworden zu sein, ich gehöre auch daحu... Was dich angeht, Du vernachlässigst deine LEBENSERINNERUNGEN zu schreiben und sie zu publizieren, Du veräggerst sie. Bevor die Themen obsolet werden, solltest Du diese Lebenserinnerungen schnell zu ende schreiben und veröffentlichen. Wenn Du das nicht machst, wirst Du deiner historischen Mission nicht gerecht werden und ein großes Verbrechen begangen und einen großen Verlust erlitten haben... Oh, verehrte LeserInnen, die redlich sind und Ihr ganzes Vermögen unserer Türkei, die ein Garten Eden ist, gestiftet haben, oh, Sie alle, die Ehl-i Beyt (Die Familie des Propheten Muhammed und des Imam Ali und seiner Nachkommen) aus dem ganzen Herzen lieben! Ich habe meine vorliegenden bescheidenen Lebenserinnerungen nicht mit der Absicht geschrieben, irgend jemanden feindlich zu behandeln. Ich habe sie aber wohl mit der Absicht geschrieben, damit wir Aleviten, Sunniten zusammen „EINSWERDEN, GROSS WERDEN, LEBENDIG WERDEN; glücklich und friedlich werden; damit wir unsere eine Scheibe Brot in Rube essen können, damit wir lieben und geliebt werden; damit wir nicht zu den Völkern, die auf der Höhe der Zeit sind, hinterherschauen, damit wir aufsteigen, leben und leben lassen... "Wenn Sie Fehler und Mangel von mir sehen, bitte verzeihen Sie diese. Jetzt lasse ich Sie mit meinen Lebenserinnerungen alleine.“

Da die Themen in dem Buch sehr umfassend behandelt wurden, wurden hier nur wichtige Sachverhalte in ihren Grundzügen hervorgehoben. Eine gründliche Auseinandersetzung mit den Lebenserinnerungen von Yaman Dede macht deutlich, dass das Verständnis der Religionspolitiken, die in der Türkei von der Osmanischen Periode bis in die republikanische Zeit und bis heute verfolgt wurden, am besten 
auf der Grundlage direkter Beobachtung möglich sind. In diesem Sinne enthalten die Lebenserinnerungen von Mehmet Dede sehr wichtige Informationen für ForscherInnen bereit, die auf dem Gebiet des Alevitentum-Bektaschitum arbeiten.

Unser Respekt und Dankbarkeit gilt allen, allen voran an Mehmet Yaman Dede, dessen Erlebnisse die Menschheit beleuchten werden, und allen geistigen Oberhäuptern (pirler) und Strebenden (talipler), die die Menschenliebe und die Liebe zur Familie des Propheten Muhammed und des Imam Ali (ehl-i beyt) als Fundament ihres Glaubens ansehen und sich Verdienste bei der Übermittlung des Alevitisch-Bektaschi Weges bis in die Gegenwart hinein erworben haben. Wir sind der Überzeugung, dass dieses vorliegende Buch großen Nutzen, allen voran für AkademikerInnen sowie für alle LeserInnen stiften wird.r 